\begin{tabular}{|c|l|}
\hline Title & $\begin{array}{l}\text { Neural mechanism for generating and switching motor patterns of rhythmic movements of ovipositor valves in the } \\
\text { cricket }\end{array}$ \\
\hline Author(s) & Ogawa, Hiroto; Kagaya, Katsushi; Saito, Mitsuo; Y amaguchi, Tsuneo \\
\hline Citation & $\begin{array}{l}\text { Journal of Insect Physiology, 57(2), 326-338 } \\
\text { https://doi.org/10.1016/.jinsphys.2010.11.021 }\end{array}$ \\
\hline Issue Date & 2011-02 \\
\hline Doc URL & http://hdl.handle.net/2115/47376 \\
\hline Type & article(author version) \\
\hline File Information & JIP57-2_326-338.pdf \\
\hline
\end{tabular}

Instructions for use 
Ms. Ref. No.: IP-D-10-00208R1

\section{Neural mechanism for generating and switching motor patterns of rhythmic movements of ovipositor valves in the cricket.}

Hiroto Ogawa $^{1,2 *}$, Katsushi Kagaya ${ }^{2}$, Mitsuo Saito ${ }^{1}$ and Tsuneo Yamaguchi ${ }^{1}$

1 Department of Biology, Faculty of Science, Okayama University, Okayama 700-8530, Japan

2 Department of Biological Sciences, Faculty of Science, Hokkaido University, Sapporo 060-0810, Japan

Abbreviated title: Oviposition motor control of cricket

Discipline: Neurobiology

*Correspondence to: Hiroto Ogawa

Department of Biological Sciences, Faculty of Science, Hokkaido University

Kita 10-jyo, Nishi 8, Kita-ku, Sapporo 060-0810, Japan

Phone/Fax: +81-11-706-3525

E-mail: hogawa@sci.hokudai.ac.jp 


\section{ABSTRACT}

In adult female crickets (Gryllus bimaculatus), rhythmic movements of ovipositor valves are produced by contractions of a set of ovipositor muscles that mediate egg-laying behavior. Recordings from implanted wire electrodes in the ovipositor muscles of freely-moving crickets revealed sequential changes in the temporal pattern of motor activity that corresponded to shifts between behavioral steps: penetration of the ovipositor into a substrate, deposition of eggs, and withdrawal of the ovipositor from the substrate. We aimed in this study to illustrate the neuronal organization producing these motor patterns and the pattern-switching mechanism during the behavioral sequence. Firstly, we obtained intracellular recordings in tethered preparations, and identified 12 types of interneurons that were involved in the rhythmic activity of the ovipositor muscles. These interneurons fell into two classes: "initiator interneurons' in which excitation preceded the rhythmic contractions of ovipositor muscles, and 'oscillator interneurons' in which the rhythmic oscillation and spike bursting occurred in sync with the oviposition motor rhythm. One of the oscillator interneurons exhibited different depolarization patterns in the penetration and deposition motor rhythms. It is likely that some of the oscillator interneurons are involved in producing different oviposition motor patterns. Secondly, we analyzed oviposition motor patterns when the mecahnosensory hairs located on the inside surface of the dorsal ovipositor valves were removed. In deafferented preparations, the sequential change from deposition to withdrawal did not occur. Therefore, the switching from deposition pattern to withdrawal pattern is signaled by the hair sensilla that detect the passage of an egg just before it is expelled. 
Key words: ovipositor; motor pattern; central pattern generator; interneuron; proprioceptor; cricket 


\section{INTRODUCTION}

Egg-laying procedures widely vary among insects (Engelmann, 1970). Some species of Orthoptera display adaptive oviposition behavior, which includes searching a suitable oviposition site, digging soil, penetrating their ovipositor and depositing eggs. In the oviposition behavior of the cricket, several sequential steps are also observed (Dambach and Igelmund, 1983; Sugawara and Loher, 1986). In the searching step, a female cricket placed on a substrate immediately starts examining the surface with her antenna, and taps the ground with the maxillary and labial palpi while walking forward. Soon after locating a suitable site, the cricket adopts an oblique position by elevating her body on her hindlegs. The cricket then uses the tip of her ovipositor to penetrate the substrate, often to its entire length when soil conditions are right. This is the penetration step of the behavioral sequence. The duration of this penetration step is about 20-25 seconds. After a short lift of the abdomen, followed by a brief rest phase (6-9 seconds), an egg appears at the base of the ovipositor and is pushed distally through the ovipositor canal. When the egg is passed to the distal part of the ovipositor, the female pulls the ovipositor out of the substrate for a short distance equivalent to the length of the egg. These alternating deposition and withdrawal steps are then repeated sequentially 5-7 times, without intervening intervals, leaving a column of 5-7 eggs along the vertical shaft dug by the ovipositor. Durations of the egg-deposition and the withdrawal are 3-10 seconds and 10-13 seconds respectively.

In this way, the ovipositor valves are used as an important effector organ through the cricket egg-laying sequence. The cricket ovipositor consists of two pairs of long valves. The dorsal valves (valve 3) are connected with the ventral pair (valve 1) by a tongue-and-groove joint. The ridges on the dorsal valve can slide freely in the grooves of the ventral valve (Snodgrass, 1933; Matsuda, 1976). Rhythmic sliding movements of 
the valves are observed in the penetration, egg-deposition and withdrawal steps during the oviposition behavior. It has been confirmed that the central pattern generator (CPG) concept (Delcomyn, 1980) is applied to the movements of ovipositors in insects. For example, electrical recordings from the ovipositor nerves in the isolated nervous system of female grasshoppers showed spontaneous rhythmic bursting activity (Thompson, 1986a). Pharmacological block of descending inhibition initiated rhythmic activity produced by the ovipositor CPG in the 7 th and 8th abdominal ganglia in the locust (Facciporte and Lange, 1996). It has also been reported that in the cricket the movement patterns of the ovipositor in crickets are preprogrammed in the central nervous system (Carrow et al., 1982). However, the motor programs for the rhythmic sliding of ovipositor valves have been not analyzed quantitatively among the different steps of the cricket oviposition behavior. Our recording of electromyogram (EMG) from the ovipositor muscles in freely-moving cricket indicated that the temporal pattern of motor activity was sequentially changed, corresponding to the shifts between behavioral steps. The main purpose of this study is to determine a neuronal architecture generating these multiple motor patterns for the rhythmic sliding of the ovipositor valves.

To generate the multiple motor patterns, distinct anatomical circuits of neurons can operate in more than one stable mode, and their connectivity can switch between functional activity patterns under various conditions. These polymorphic neuronal circuits have been referred to as 'reorganizing circuits' (Morton and Chiel, 1994) or 'multifunctional circuitry' (Kristan et al., 1988). Most of studies of the multifunctional circuitry have focused on rhythmic movements and their underlying CPG (Grillner, 2006; Kiehn, 2006; Marder, 2000; Marder and Bucher, 2001). Briggman and Kristan (2008) have classified the multifunctional circuits into five basic architectures. They also proposed which two types of neural organization of multifunctional circuitry, that 
were 'dedicated unifunctional neuron pools' and 'multifunctional neuron pools'. In this study, to reveal which types of multifunctional circuit are applicable to the CPG for rhythmic sliding of the cricket ovipositor valves, the interneurons that are involved in the rhythmic activity of the ovipositor muscles were studied with intracellular recording from the terminal abdominal ganglion (TAG). Furthermore, we examined the mechanosensory hairs that are located on the inside surface of the dorsal ovipositor valves, and that are likely to provide afferent signals for switching the oviposition motor patterns adjusting to the sequential steps in egg-laying behavior. 


\section{MATERIAL AND METHODS}

\subsection{Animals}

Crickets (Gryllus bimaculatus) were reared in the laboratory under a 12-12 $\mathrm{hr}$ light-dark cycle at a constant temperature of $28^{\circ} \mathrm{C}$. For all experiments, females were used that were 12-14 days past the imaginal molt.

\subsection{Anatomy and morphology}

Precise location and attachments of ovipositor muscles and cuticular plates were observed under a stereomicroscope, and determined using Snodgrass (1933) as a general guide. The movement that would be produced by muscle shortening was then deduced. The innervation of muscles was studied in dissections stained with methylene blue (Wako Pure Chemical, Osaka, Japan). The motor neurons were stained by retrograde filling with $200-250 \mathrm{mM} \mathrm{NiCl} 2$ for $18-48 \mathrm{hr}$ at $4^{\circ} \mathrm{C}$ from the proximal cut end of the nerve which innervated each ovipositor muscle. The nickel ions were then precipitated within the neurons by addition of rubeanic acid (Wako) to the cricket saline ( $\mathrm{NaCl} 150 \mathrm{mM}, \mathrm{KCl} 9 \mathrm{mM}, \mathrm{CaCl} 25 \mathrm{mM}$, NaHCO $32 \mathrm{mM}$, Dextrose $50 \mathrm{mM}$, Trizma $\mathrm{HCl} 40 \mathrm{mM}$, Trizma Base $0.01 \mathrm{mM}, \mathrm{pH} 7.2$, Wako). After fixation with 10\% formalin, the ganglion was dehydrated and cleared for whole mount viewing. Usually, the ganglion stained with the nickel ion was intensified, following the method of Bacon and Altman (1977). The precise distribution and external structure of the hair sensilla on the ovipositor valves were examined with a scanning electron microscope (T-300, JOEL, Tokyo, Japan) in the isolated valves that had been fixed with acetone (Wako), dehydrated, $\mathrm{CO} 2$-critical-point dried and then coated with gold.

\subsection{EMG recordings in freely-moving animals}


Crickets were anesthetized by placement in a cooling room $\left(4^{\circ} \mathrm{C}\right)$ for $20 \mathrm{~min}$. Small holes $(\varnothing=0.5 \mathrm{~mm})$ were made on both lateral sides of the 9th tergum, and a pair of electro-polished tungsten-wire electrodes $(\varnothing=50 \mu \mathrm{m})$ were inserted into the ovipositor muscles through the holes. A copper wire $(\varnothing=50 \mu \mathrm{m})$, to be used as a reference electrode, was inserted into the dorsal side of the abdomen. The incisions were covered with wax. The operated animal was put on wet cotton as a substrate for oviposition, and electromyograms (EMGs) were recorded during the oviposition behavior. After EMG recording, the abdomen was dissected to confirm the identity of the recorded muscles according to location of the wire electrodes.

\subsection{Electrophysiology in tethered preparation}

Crickets were pinned dorsal side up on a paraffin platform after removing the wings and legs. An incision was made along the dorsal midline of the abdomen, and the gut, internal reproductive organs (except for the genital chamber) and surrounding fat were removed. This exposed the TAG and the muscles whose contraction generate the movements of the ovipositor valves. In the preparations for intracellular recording, a piece of filter paper soaked into $1 \%$ protease (type XIV, Sigma-Aldrich, St. Louis, MO) was put onto the TAG for $5 \mathrm{~min}$, in order to soften the sheath to enable insertion of a glass microelectrode. The preparation was rinsed with cricket saline to quench the protease from continuing its effect on the TAG. A stainless-steel spoon was introduced posteriorly-directed between the 8th lateral nerves to hold the TAG, and also served as an indifferent electrode. The TAG was held within a ring made of tungsten wire, so that it was immobile during the rhythmic movements of the ovipositor. To eliminate sensory feedback from the mechanosensory hairs on the cerci, they were coated with vaseline. 
For recordings of EMGs, electro-polished tungsten wire $(\varnothing=70 \mu \mathrm{m})$ was inserted into the ovipositor muscles. To elicit the rhythmic activity of the ovipositor muscles in the tethered preparation, the connective nerve cords were cut between the 6th and terminal abdominal ganglia. In cases where the nerve-cord cutting did not induce the rhythmic activity, tactile stimulus to the tip of the ovipositor valves or application of 1-mM dopamine into the abdominal cavity were used.

For extracellular recording from sensory neurons, a part of the cuticle of the dorsal ovipositor valve was removed. The sensory nerve cord was exposed, and a bipolar hook electrode made of tungsten wire $(\varnothing=50 \mu \mathrm{m})$ was positioned under the nerve cord. The manual tactile stimulus was applied to hair sensilla on the ovipositor valves using a small stainless-steel spatula connected to the probe of a micromanipulator.

For intracellular recording and staining of interneurons, a glass microelectrode filled with $1-\mathrm{M} \mathrm{LiCl}_{2}$ containing 4\% (W/V) Lucifer Yellow (Sigma-Aldrich) was used. After physiological recording, Lucifer Yellow was iontophoretically injected into an interneuron with a hyperpolarizing current of $3 \mathrm{nA}$ for 1-3 min. Then, the TAG containing the interneuron was isolated, fixed in $4 \%$ formaldehyde in a $0.2-\mathrm{M}$ acetate buffer ( $\mathrm{pH} 4.3$ ) for up to $20 \mathrm{~min}$, dehydrated in ethanol and cleared in methylsalicylate. Stained interneurons were drawn in whole mount using a camera lucida under a fluorescent microscope (BH-RFL, Olympus, Tokyo, Japan) in combination with an excitation filter (BG-12, Olympus).

\subsection{Statistical analysis}

For statistical analysis of the oviposition motor patterns, we used $\mathrm{R}$ statistical software (ver. 2.11.0, R Development Core Team, http://www.R-project.org) and performed generalized linear modeling (GLM), which is a methodology developed by 
extending the linear model (Faraway, 2006). In this study, we need statistical models representing the oviposition motor patterns to compare them between three behavioral steps and to categorize the motor pattern evoked in the tethered preparation for intracellular recording method. Then, we used an extended linear model assuming that the burst duration increased linearly as the cycle period increased. In this model, the distribution around the mean of the burst duration did not follow a normal distribution as in the standard linear regression, but a gamma distribution. This is because the variability does not seem equal as assumed in normal linear regression model, but seems increasing depending on the increase of cycle period. Therefore, we considered the burst duration as a response variable and the cycle period as predictor variables,

(burst duration $)=b_{0}+b_{1} x($ cycle period $) \ldots(1)$

where $b_{0}$ and $b_{1}$ are parameters of linear predictors to be estimated. The variability was also estimated as a dispersion parameter using the function gamma.shape() in the package MASS (Venables and Ripley, 2002). At first, we considered two models: the model (1)-I (in the equation (1), $b_{0} \neq 0$ and $b_{1} \neq 0$, both were to be estimated) and model (1)-II (in the equation (1), $b_{0} \neq 0$ was to be estimated and $b_{1}=0$ ). We confirmed whether the model (1)-I is better than the model (1)-II for all behavioral steps. To select a better model, we adopted Akaike information criterion (AIC), which can be expressed as follows: $\mathrm{AIC}=-2 \log L+2 k$, where $L$ is the maximum $\log$-likelihood and $k$ is the number of parameters involved in the model (Akaike, 1974). AIC is one of generally used criteria in the problem of model selection when we choose a better model among multiple statistical models. It can be interpreted that when we have multiple models that give almost the same maximum log-likelihood values, AIC point out that we should 
select the model using minimum number of parameters. Calculation of the AIC value was performed by the $\operatorname{glm}()$ function of the R package.

To confirm whether the activity pattern of muscle M217 sufficiently represents the three behavioral steps that are penetration, egg-deposition and withdrawal, we compare a model,

(burst duration $)=b_{0}+b_{1} \times($ cycle period $)+b 2 \times($ behavioral step $) \ldots$ (2)

with the model (1) in terms of AIC. If the model (2) is better, we have to take into account the behavioral step and the activity pattern of M271 sufficiently represents the behavioral steps. For the further assessment of the difference among three motor patterns, we performed a model selection among following three types of model (1). The first is 'three regression-lines model', which gives three regression lines for the motor patterns of each behavioral step, penetration, deposition and withdrawal. The second is 'two regression-lines model' giving two lines, one of which is for one category taking together two behavioral steps and the other of which is for the other step. The third is 'single regression-line model' which gives one regression line for all behavioral steps. If the three regression-lines model is better than any other two regression-lines model or single regression-line model, three behavioral steps can be considered different each other.

To categorize a focal motor pattern evoked in the tethered preparation to any one of three behavioral steps, we applied the GLM in two types of analysis. In the first analysis, we construct new regression-line models for relationship between the cycle period and the burst duration, in which the data set include the data of the newly-obtained motor patterns in addition to previous data measured in each behavioral step, penetration, 
deposition or withdrawal. Next, we compared the sum of AIC values of the three models. If a model in which new data were added to the data set of a specific behavioral step gives the smaller value of AIC than the other two models, it means that the motor patterns can be categorized to the specific behavioral step. In the other analysis, we considered the difference in the distribution of the cycle period. Here, we performed GLM to the distribution of the cycle period as the model described below,

$$
\text { (cycle period })=b_{0}+b_{1} x(\text { behavioral step) } \ldots(3)
$$

and compared which of models: the model $(3)-\mathrm{I}\left(b_{0} \neq 0, b_{1} \neq 0\right)$ and the model (3)-II $\left(b_{0} \neq 0\right.$, $\left.\mathrm{b}_{1}=0\right)$ is better in terms of AIC value. According to these two analyses, we assessed the validities of the categorization of newly obtained motor patterns. 


\section{RESULTS}

\subsection{Ovipositor muscles and their innervations}

The ovipositor valves are connected to the gonapophyses, which are internal apodemes projecting antero-laterally into the abdominal cavity. Dorsal and ventral gonapophyses are hinged together at their bases, and each gonapophysis is hinged with the 8th or 9th sternum. The ovipositor is operated by five pairs of muscles, as shown in Fig. 1A. Four of the pairs (M271, M273, M285 and M288) are attached to the dorsal gonapophysis, and the fifth pair of muscles (M272) is attached to the ventral gonapophysis. Contraction of these muscles pulled or swings the gonapophyses, and their movements are transmitted to the ovipositor valves. Forward-and-backward movement of the dorsal valve is produced by alternate contractions of a protractor, M288, and a retractor, M271. The contraction of M288 pulls the dorso-lateral end of the apodemes toward their attachment on the 8th tergum, and rotates the dorsal gonapophysis around its connection with the tergum (Fig. 1B). This movement in turn gives the dorsal valve itself a backward movement. The dorsal valve is then retracted by the contraction of M271, which is attached to the ventral end of the apodemes near their connection with the valve, and the gonapophysis returned to the rest position. M273 and M285 also protract the dorsal valve, and M272 which is segmentally homologous to M271 retracts the ventral valve. In this study, we could not identify a protractor muscle of the ventral valve.

The ovipositor muscles are innervated by a few ventral segmental nerves of the TAG (Fig. 2A), which emerge from the fused ganglia of the 6th to 11th abdominal segments (Jacobs and Murphey, 1987). The ventral root of the ninth segmental nerve (9vpn) supplies the muscles operating the dorsal valve. It extends branches ventrally to the four muscles, M284, M285, M288 and M289, and to the genital chamber. The lateral branch 
of 9vpn supplies two muscles, M271 and M273, and the main branch entered the dorsal valve. The muscle M272 operating the ventral valve is innervated by a lateral branch of the ventral root of the eighth segmental nerve (8vpn).

The central locations of motoneurons supplying M271, M273 and M272 were determined by retrograde staining with nickel chloride of each branch of $8 \mathrm{vpn}$ or $9 \mathrm{vpn}$ innervating these ovipositor muscles (Fig. 2B). We have stained motoneurons in 10 preparations for backfilling of $8 \mathrm{vpn}$ and $9 \mathrm{vpn}$ respectively. But there are variations in the number of the stained cells because of uncertainty of the method. Further, we did not succeed in backfill-staining of the other branches innervating M285 and M288. Fig. 2B shows direct drawings of the best samples in which a larger number of motoneurons were clearly stained than that in other preparations. Unfortunately, it was difficult to stain the motoneurons supplying M271 and M273 separately. These muscles are innervated by 10 motoneurons with somata having diameters of 30 to $70 \mu \mathrm{m}$ on the ipsi-lateral side of the TAG (indicated by arrowheads in Fig. 2B1). Their cell bodies are located in three clusters that includes a dorsal group of cell \#1-4, a middle group of cell \#5-9 and a ventral group of cell \#10 as shown in the lateral view of the TAG (Fig. 2B1). Backfill staining of the branch of 8vpn to M272 revealed four cell bodies having diameters of 20 to $50 \mu \mathrm{m}$, which are located antero-laterally in a cluster near the ventral surface of the TAG by the emergence of 8vpn (Fig. 2B2). The dendrites of the ovipositor motoneurons arborize near the dorsal surface of the TAG, but do not extend to contralateral hemisphere of the ganglion.

\subsection{Motor program of ovipositor movement}

At first, we performed EMG recordings from the ovipositor muscles in tethered and dissected preparation. In this preparation, oviposition motor patterns rarely occurred 
spontaneously, unlike with the free-moving preparation. We used three kinds of stimulation to elicit the rhythmic activity of the ovipositor muscles: transaction of the connective nerve cords, tactile stimulation to the tip of the ovipositor valves and bath application of 1-mM dopamine. Figure 3 shows EMGs recorded from different pairs of ovipositor muscles (Fig. 3A), and the predicted motor program (Fig. 3B). During rhythmic contractions, right and left homologous muscles, for example M271R and M271L, were activated in antiphase, and right and left ovipositor valves were protracted/retracted alternately. M273, M285 and M288 which are antagonistic muscles of M271 were activated in antiphase with ipsilateral M271. Further, M272 also contracted in antiphase with M271. It suggests that the dorsal and ventral ovipositor valves slide alternately during oviposition motor rhythm. The oviposition motor pattern evoked in the tethered preparation was, however, unstable and varied in their rhythm between recorded animals. Then, we used implanted wire-electrodes for EMG recording in free-moving cricket, which carry on the egg-laying behavior.

Sexually mature female crickets that are placed on the moist substrate spontaneously begin oviposition behavior. EMG recordings from the ovipositor muscles during the sequence of the oviposition behavior showed stable rhythmic motor patterns in the penetration, egg-deposition and withdrawal steps (Fig. 4A). Here, we focused activity of the retractor M271, because the EMG of this muscle could be easily recorded by the implanted wire-electrode in freely-moving cricket and exhibited distinct rhythms as described later. Actually, EMG recording from M271 are sufficient to distinguish the motor patterns among three behavioral steps. To compare these motor patterns, we measured the cycle period of successive electrical discharges and the duration of each discharge of M271 from EMG recorded at different behavioral steps. Table 1 indicates mean values and standard deviations of the cycle period and the burst duration for three 
motor patterns. These parameters are components constituting the rhythmic motor activity, and give the motor patterns a distinction. Further, we analyzed the relationships between the cycle period and the burst duration. This approach is a conventional method for comparing the motor patterns in detail. For example, it was used to examine how deafferentation could affect the flight motor pattern in locust (Pearson and Wolf, 1987; Stevenson and Kutsch, 1987). The relationships between the cycle period and the burst duration showed significant interdependence in all three motor patterns respectively, as indicated by AIC values between the model (1)-I and the model (1)-II (see, Materials and Methods). The AIC values of the model (1)-I were all smaller than the model (1)-II for each behavioral steps, suggesting the interdependence between the cycle period and the burst duration (Table 1).

Next, to confirm whether the relationship between the cycle period and the burst duration of M217 sufficiently represents the three behavioral steps, we compared the model (2) which includes the predictor variable of behavioral step, with the model (1) which does not include the variable. The AIC value of the model (2) was 2323, and the model (1) was 2505. Thus, we have to take into account the behavioral step and the motor pattern of M217 sufficiently represents the three behavioral steps. Figure 4B plots the cycle period versus the burst duration for each oviposition motor pattern in 6 preparations, indicating linear relationships between the cycle period and the burst duration for all three motor patterns. The equations with the predicted parameters are shown in the Table 1. The sum of AIC values of the three regression-lines model (see Materials and Methods) was 2303.8, which is smaller than that of the single-line model, 2505.0. Compared with the two regression-lines models in the sum of their AIC values (penetration and the others: 2328.3, withdrawal and the others: 2344.2, deposition and the others: 2445.6), the three regression-lines model is smallest in the sum of AIC value 
among all models. These results indicate that EMGs recorded during the egg-deposition, penetration and withdrawal steps exhibit different relationships between the cycle period and the burst duration, meaning that the motor patterns for three behavioral steps differ from each other in the relative duration of a discharge to the inter-burst-interval. Hence, we used following two references for distinction of the motor patterns: the first is the linear regression model, and the second is the distribution of the cycle period.

\subsection{Activity patterns of interneurons mediating the oviposition motor pattern} generation

There are two prominent types of architecture for multifunctional circuit generating multiple motor patterns (Briggman and Kristan, 2008). The first is dedicated unifunctional neuron pools, each of which controls distinct motor patterns and usually inhibit each other. The other is a multifunctional neuron pool, which controls multiple motor patterns occurring in mutually exclusive behaviors. To describe the neural organization for generating the multiple oviposition motor patterns, we recorded the activities of 53 interneurons during rhythmic movement of the ovipositor in tethered preparations. As described above, the oviposition motor patterns forcibly-induced in the tethered preparation were unstable, and the stimulations could evoke any one of the three types of motor pattern. Therefore, using the GLM in two types of analysis, we categorized a focal motor pattern evoked in the tethered preparation to any one of three behavioral steps (see Materials and Methods).

We identified 12 types of interneurons that were involved in the rhythmic activity of the ovipositor muscles within the TAG (Five types of them were described in the result section of this paper, the others were shown in supplemental figures). These interneurons included local spiking interneurons (LSI) within the TAG and non-giant 
interneurons (NGI) having axons projecting into the connective nerve cord. The resting potential of these spiking interneurons was about $-45 \mathrm{mV}$. This value corresponded to that of the wind-sensitive spiking interneurons identified within the cricket TAG (Baba et al., 1991, 1995). All identified interneurons were named in accordance with conventions established in these previous studies. These ovipositor interneurons fell into two classes: 'initiator interneurons' and 'oscillator interneurons'. Spontaneous action potentials were observed in both types of ovipositor interneurons, but their firing frequency varied in different neurons.

Excitation of the 'initiator interneurons' preceded the rhythmic contractions for ovipositor muscles. We found three such initiator interneurons. One of these was a local interneuron 8V-1 (LSI-8V-1), shown in right drawing of Fig. 5A. The frequency of spontaneous spikes in this cell increased during rhythmic activity of M271 (Fig. 5A1). This frequency increase in spontaneous firing was observed whenever M271 showed rhythmic activity, which was recorded 6 times in the preparation shown in Fig. 5A. When its spontaneous firing was suppressed by hyperpolarizing current injection of $-1 \mathrm{nA}$, action potentials preceded the initiation of the rhythmic discharges for M271, and the delay from firing of LSI-8V-1 to the first discharge of M271 was about 90-140 ms. The rhythmic activity of EMG stopped following termination of the firing in this interneuron (Fig. 5A2). The cycle period $(132.3 \pm 17.6$, mean \pm SD in $\mathrm{ms})$ and the burst duration $(46.0 \pm 8.7)$ of the M271 activity in rhythmic contraction induced by firing of LSI-8V-1 are similar to those in the deposition pattern. In another local interneuron, LSI-8L-2, we also observed that the increase in spontaneous firing preceded the rhythmic activity in M271, and that hyperpolarizing current injection into this neuron completely suppressed the ovipositor motor pattern generation (Supplemental Fig. 1). 
Further, depolarizing current injection of $+1 \mathrm{nA}$ into another 'initiator interneuron', the non-giant interneuron 17 (NGI-17) shown in right drawing of Fig. 5B, was capable to induce rhythmic activity in M271 (Fig. 5B). This induction of M 271 activity was observed 3 times in this preparation. NGI-17 evoked action potentials at about $50-\mathrm{Hz}$ frequency during the rhythmic activity of M271, while the frequency of spontaneous firing that induced little rhythmic motor activity was less than $20 \mathrm{~Hz}$. Both cycle period $(104.8 \pm 22.3)$ and burst duration $(30.3 \pm 11.1)$ of the rhythmic discharge is shorter than those of the deposition pattern.

We performed GLM for the motor pattern induced by activation of the interneurons (Fig. 5C). Further, we compared the AIC values of the new models to the three motor patterns in order to categorize the motor patterns induced (see Materials and Methods). When the data of the motor patterns induced by firing of LSI-8V-1 were incorporated into the 'penetration' data set, the AIC value of new model was 2383.2. The AIC value of the new model for deposition was 2381.6, and that for withdrawal was 2393.2. Thus, the model for deposition gave the smallest value. In the same way, the AIC values of new models obtained from data of the motor pattern evoked by current injection into NGI-17 were 2369.5 for penetration, 2368.5 for deposition and 2380.1 for withdrawal, respectively, meaning that the motor pattern fits with the deposition motor pattern in terms of AIC. These results suggested that LSI-8V-1 and NGI-17 play roles in initiating the egg-deposition pattern.

In the 'oscillator interneurons,' rhythmic oscillations of membrane potential and rhythmical bursting activity were in phase with the oviposition motor rhythm. Nine types of oscillator interneurons including 3 types of ascending interneurons and 6 types of local interneurons were found in the TAG (Supplemental Fig. 2). In most of them, even though their rhythmic bursting was suppressed by a hyperpolarizing current 
injection, there was little influence on the EMG patterns of ovipositor muscles. It is likely, therefore, that these interneurons are neither necessary nor sufficient for the oviposition pattern generation. However, the local interneuron 9L-4 (LSI-9L-4) had an effect on one muscle. LSI-9L-4 normally exhibited rhythmic bursts synchronized with ovipositor muscles (Fig. 6A), and suppression of that cell's activity inhibited the rhythmic activity in M271 (Fig. 6B). This may indicate that LSI-9L-4 generates rhythmic outputs to the motoneurons driving M271. Even while action potentials were suppressed by the hyperpolarizing current injection, the rhythmic depolarization was still observed in LSI-9L-4 (upward arrowheads in Fig. 6B).

Two types of cells in the class of 'oscillator interneuron' showed different activity patterns from the others: these two cells exhibited biphasic depolarization phase-locked with the discharges of the ovipositor muscles. The cell shown in the right drawing of Fig. 7A has been identified as local interneuron 10-2 (LSI-10-2, Baba et al., 1995). The LSI-10-2 represented the rhythmic depolarization and bursting of short duration that was monophase-locked with the discharge of M271 at higher frequencies of the oviposition motor rhythm (Fig. 7A1). At lower frequency rhythms, two components could be distinguished in the rhythmic depolarization; the early component preceded each discharge of the ipsi-lateral M271 and the later component followed that discharge (upward arrowhead in Fig. 7A2). There was another oscillator interneuron that exhibited a similar biphasic depolarization pattern: local interneuron 9V-4 (LSI-9V-4), shown in the right drawing of Fig. 7B. These findings demonstrated that some types of oscillator interneurons could exhibit biphasic oscillation during the ovipositor motor pattern in slower rhythm.

We analyzed the relationship between cycle period and burst duration in EMG recorded during periods when monophasic- or biphasic depolarizations were observed 
in LSI-10-2 (Fig. 7C). We calculated the AIC values for the EMGs observed with monophasic- or biphasic depolarization in addition to each data-set for three motor patterns. The AIC values given for the biphasic pattern were 2391.0 for penetration, 2422.1 for deposition and 2468.5 for withdrawal, respectively, meaning that the motor pattern observed with biphasic depolarization is categorized to penetration. The values for the monophasic pattern were 2386.2 for penetration, 2436.9 for deposition and 2443.0 for withdrawal. Thus, the penetration model also gave the smallest value. However, the cycle period in the motor pattern observed with monophasic depolarization was $151.8 \pm 22.2 \mathrm{~ms}$, which was similar to that of the deposition pattern rather than penetration pattern. The GLMs for relationship between the cycle period and the burst duration do not take into account the distribution of the cycle period. We, therefore, performed GLM for the distribution of the cycle period to categorize the monophasic motor pattern. In this analysis, we compared two types of models for each behavioral step (Fig. 7D). The first is the 'one distribution model', in which it was assumed that the two data-sets which include the newly-added data (shown in upper plots in each panel of Fig. 7C) and the previous data in free-moving preparation (shown in lower plots) were given from one distribution (solid curves in Fig. 7D). The second is the 'two distribution model', in which it was assumed that these data-sets were given from different two distributions (broken curves in Fig. 7D). AIC values of these models given for penetration were 1165.7 (one distribution) and 1120.4 (two distribution), respectively. Those for deposition were 1098.0 (one distribution) and 1099.5 (two distribution), and for withdrawal were 1023.4 (one distribution) and 965.62 (two distribution). Thus, only in the case of deposition, smaller AIC value was given for one distribution model, meaning that the motor pattern observed with monophasic depolarization was categorized to egg-deposition pattern. This result suggests that the 
monophasic depolarizations occurred during the egg-deposition motor pattern, and that the biphasic depolarizations occurred during the penetration motor pattern. It was supposed that LSI-10-2 could receive different patterns of synaptic inputs between the egg-deposition motor pattern and penetration motor pattern.

\subsection{Hair plates sensilla for switching of ovipositor motor patterns}

Electromyograms recorded in freely-moving crickets showed that the egg-deposition motor pattern was converted into the withdrawal pattern instantaneously without a pause, rather than gradually. Sensory signals for this switching in ovipositor motor patterns appear to be provided by mechanoreceptive organs. In order to examine central projection of sensory neurons in the TAG, afferent fibers from ovipositor valves were stained via back-filling with nickel chloride (Fig. 8A). The axons arborized in the ipsilateral field of the 8th or 9th neuromere and at a more antero-medial field in the 7 th neuromere of TAG. Their branches were confined to the ipsilateral hemisphere of the TAG. Some afferent fibers from the ovipositor valve projected to the 6th abdominal ganglion through the connective nerve cord. Scanning electron microscopic observation revealed two groups of hair sensilla distributed on the inner side of the tip of the dorsal valve in Gryllus bimaculatus (Fig. 8B). The proximal group was a cluster of 4-7 hairs whose shafts were entirely smooth and comparatively short, 10-25 $\mu \mathrm{m}$ in length (right panel of Fig. 8C). The other group was a row of 11-15 larger hairs whose shafts were slightly curved distal-ward. Their shaft lengths varied from 20 to $70 \mu \mathrm{m}$, and gradually decreased from the proximal side toward the distal end of the valve (left and center panel of Fig. 8C).

Extracelluar recording from sensory afferents showed tonic responses to deflection of these hairs (Fig. 9A). Posterior-ward deflections evoked discharges with larger 
spikes than those evoked by stimuli in the anterior direction. When the rows of hair sensilla were removed, or when the afferent nerves were cut at the base of the dorsal valves in freely-moving preparation, the deposition step was not switched to the withdrawal step; the deposition motor pattern persisted even after an egg was expelled (Fig. 9B). This deafferented cricket, therefore, messed up the eggs deposited around the hole. In an intact cricket, the ovipositor was pulled out of the substrate for a short distance through the withdrawal step previous to the next egg appear at the base of the ovipositor so that the eggs could be laid in tandem within an hole of substrate. Actually, the withdrawal motor pattern was stopped at the same time as the egg was expelled from ovipositor valves in the intact cricket (Fig. 9B1). These results lead the following hypothesis: in the deposition behavior, passage of an egg along the ovipositor could deflect the mechanosensory hairs posteriorward just before egg is expelled, and the switch of the motor pattern from deposition to withdrawal could be triggered by the afferent signal from the hair plates detecting the egg passage. 


\section{DISCUSSION}

\subsection{Distinct motor patterns of the ovipositor valves}

The sequential steps of oviposition behavior have been described in detail for $G$. bimaculatus (Dambach and Igelmund, 1983) and T. commodus (Sugawara and Loher, 1986). Through these observations, it was demonstrated that rhythmic movements of the ovipositor valves occur during three distinct steps: penetration, egg-deposition and withdrawal. Dambach and Igelmund (1983) cinematographed the ovipositor movements in three species of crickets, and illustrated the coordination of all valves. They found that the movement patterns varied according to the three behavioral steps. In $P$. spectrum, the ventral valves can move in unison during penetration and deposition, but not during withdrawal. In T. commodus, egg-deposition appears to be performed by the phase-shifted back-and-forth movement of every valve (Sugawara and Loher, 1986). However, motor programs of the ovipositor rhythmic movements had not been recorded and analyzed by electrophysiological techniques in freely-moving crickets, yet.

Using wire electrodes implanted into the abdomen, we recorded the electrical activity of the ovipositor muscles in freely egg-laying female crickets. The EMG recordings in the freely-moving preparations indicated that the ovipositor muscles including M288 (protractor of the dorsal valve) and M271 (retractor) altered their activity rhythms according to the behavioral steps. It was found that there are linear relationships between the cycle period and the burst duration in all three of the oviposition motor patterns. Our GLM analysis demonstrated that the models giving these relationships for the distinct patterns are different from one another. The difference in the models means that the relationship between the burst duration and the cycle period varies between the three distinct behavioral steps. This result suggests that the motor patterns for penetration, egg-deposition and withdrawal steps are not generated through modulation 
of a single pattern of motor output through sensory feedback. If these oviposition motor patterns were derived from a single motor program, the sum of AIC values of the single line model would be smallest in those of all models. In this case, the regression lines characterizing each motor pattern would have overlapped along a straight line. In the stinging motor pattern generation in the honeybee, the regression line for the relationship between the cycle period and the burst duration was constant, even when both parameters were extended substantially by sensory feedback of the mechanical stress to the sting (Ogawa et al., 1995). Therefore, the difference among the models for the relationship between the cycle period and the burst duration revealed that the motor patterns in penetration, egg-deposition and withdrawal steps were clearly different from each other, suggesting the presence of multifunctional pattern-generating circuits.

In the locust, two distinct unifunctional neuron pools control two different behaviors involved in the egg-laying behavior. The digging central pattern generator is located in the 8th abdominal ganglion, and is responsible for coordinating rhythmic movements of the ovipositor valves required for digging of the oviposition hole into which eggs are laid (Thompson, 1986a). The other pattern generator that is involved in egg-laying is located in the 7th abdominal ganglion (Facciponte and Lange, 1992). In the cricket, however, cutting the connective nerve cords between the 6th and terminal abdominal ganglia induced any one of three different kinds of rhythmic movements of ovipositor valves: penetration, egg-deposition and withdrawal. This indicates that the multifunctional pattern generator for three types of oviposition motor pattern are located within the TAG. Two distinct interneuronal circuits producing walking and flight motor patterns of locust, and two separate neuronal circuits controlling flight and stridulation in the cricket, T. commodus are located within meta- and mesothoracic ganglia (Ramirez and Pearson, 1988; Hennig, 1990). 


\subsection{Initiator interneurons for oviposition motor pattern generation}

The interneurons we recorded were classified to two main groups according to their behaviors in generation of the oviposition motor activity. We categorized LSI-8V-1, LSI-8L-2 and NGI-17 into 'initiator interneurons,' of which firing preceded on the rhythmic activity of ovipositor muscles. The rhythmic movements of ovipositors in tethered cricket was triggered by decapitation or cutting of the nerve cords. It is likely that activities of the initiator interneurons in TAG may be inhibited by descending fibers from cephalic- or thoracic ganglia, and that following release from the descending inhibitory neurons, the initiator interneurons induce the rhythmic oviposition motor programs (Fig. 10). This scheme is similar to the initiation mechanism of oviposition digging behavior in grasshopper and locust (Thompson 1986b, Facciponte and Lange, 1996). It is, however, unclear whether excitation of the single initiator interneuron is sufficient to trigger the rhythmic contraction in all ovipositor muscles, or not. Selective activation by depolarizing current injection into the identified wind-sensitive interneuron within TAG induces locomotion of the cricket (Gras and Kohstall, 1998). At least, NGI-17 was capable to induce the rhythmic activity of M271 for the egg-deposition motor pattern. On the other hand, it is possible that LSI-8V-1 and LSI-8L-2 do not contribute to initiate the oviposition motor pattern but receive common input from descending interneurons or other initiator interneurons. To make clear details of triggering mechanism of the ovipositor motor pattern generation, it would be necessary to examine responses of the initiator interneurons to release from inhibitory descending signals via the cutting of connective nerve cord or to identify that descending neurons. 


\subsection{Oscillator interneurons synchronized with ovipositor motor rhythm}

We found 9 types of 'oscillator interneurons' that exhibited rhythmic oscillation of their membrane potential or rhythmic bursting synchronized with the oviposition motor rhythms. The oscillator interneurons would be components of the oviposition CPG circuits. Particularly, LSI-9L-4 has a crucial role in generating the deposition motor pattern, because the suppression of its rhythmic bursting by a hyperpolarizing current suppressed the rhythmic contraction of M271 (Fig. 6). Unfortunately, the rhythmic oscillations observed in tethered preparations were too unstable to allow us to test a perturbation effect on a phase reset. It is, therefore, unclear whether the rhythmic depolarization in LSI-9L-4 is due to rhythmic synaptic inputs from other oscillator neurons, or is generated by endogenous oscillation of the membrane potential without synaptic inputs like 'endogenous burster neurons' (Miller and Selverston, 1982). It is possible that LSI-9L-4 is a candidate of the endogenous burster neuron for the oviposition motor pattern generation. Pharmacological experiments, for example blocking of synaptic connection with low- $\mathrm{Ca}^{2+}$ saline, would be useful for determination whether the endogenous burster neurons are included in the CPG network for oviposition motor rhythms.

\subsection{Neural circuits generating different oviposition motor patterns}

One remarkable finding in our study was that the oscillator interneuron, LSI-10-2, exhibited two distinct depolarization patterns, monophasic and biphasic depolarization, which were observed to be correlated with different oviposition motor rhythms (Fig. 7A). It was supposed that LSI-10-2 receives monophasic synaptic-input following the discharge of M271 contralateral to its soma at egg-deposition step, and that the addition of other phasic inputs preceding M271 discharges to the later monophasic 
depolarization could produce the biphasic depolarization in the penetration patterns. It is possible that LSI-9V-4 showing biphasic depolarization also receive different forms of synaptic inputs according to the behavioral steps. Interestingly, M288 exhibited two separated components of rhythmic discharge in one cycle during the penetration step, while single discharge was recorded in the deposition motor pattern (Fig. 4A). It is possible that the biphasic depolarization observed in LSI-10-2 and LSI-9V-4 could be involved in alternation of M288 motor pattern.

This variation in oscillation pattern of LSI-10-2 also demonstrated that this interneuron was shared by neuron pools for driving the deposition and penetration patterns. Like LSI-10-2, a single interneuron that changes its activity pattern dramatically between two behaviors has been found. For example, some of spinal interneuron in turtle can be rhythmically active during scratching, but tonically active during swimming (Berkowitz, 2002). Imaging study of leech segmental ganglion, some identified interneurons oscillate at very different frequencies during fictive crawling and swimming (Briggman and Kristan, 2006). Individual CPG neurons in the crab stomatogastric ganglion dynamically change their rhythmic activities and phase relation between them during pyloric rhythm, gastric rhythms or two rhythms simultaneously (Weimann et al., 1991). Probably, the pattern-generating circuits for the deposition and the penetration partly share some oscillator interneurons (Fig. 10).

Details of the CPG circuit for the oviposition motor patterns have not been determined. Since LSI-10-2 does not appear to be an endogenous oscillator for the pattern generation, the rhythmic depolarization of LSI-10-2 is most likely caused by phasic synaptic inputs from other oscillator neurons. Therefore, the existence of two distinct depolarization patterns in LSI-10-2 indicates that the number or types of interneurons from which the LSI-10-2 receives synaptic inputs are different between the 
deposition and penetration motor patterns. This fact supports the hypothesis that the neuron circuit for the oviposition motor pattern generation is rewired for the penetration and egg-deposition steps. Probably, the deposition- and penetration motor patterns will be produced by the multifunctional circuit containing oscillator interneurons some of which are dedicated oscillators and the others of which are shared between deposition and penetration neuron pools. Moreover, two kinds of oviposition pattern have not been observed simultaneously, even in tethered preparations. This suggests that the neuron pools for the different oviposition patterns are not completely independent, but share common network components. It is possible that the neuronal circuitry is dynamically reorganized for the individual behavioral steps, and is used for generation of the three different oviposition motor patterns.

\subsection{Sensory signals to switch the oviposition motor patterns}

We identified the mechanosensory hairs on the inside of the tip of the dorsal valve of G. bimaculatus. In another species of cricket, Teleogryllus commodus, the cuticle of the valves bears several types of hair sensilla on its inner and outer surface (Sellier 1971; Sugawara and Loher 1986). Two rows of large sensory hairs from which sensory input may terminate the deposition movement have been observed on the inside surface of the tip of the dorsal ovipositor valve (Sugawara and Loher 1986). We demonstrated that these hair sensilla provide a key signal to switch from the egg-deposition motor pattern to the withdrawal pattern. When afferent input from these hair-sensilla was eliminated, the deposition pattern persisted even after an egg was expelled from the ovipositor, and the next egg finally came down to the ovipositor canal without the withdrawal step. This observation suggests that the sensory signal from the hair sensilla on dorsal valve is 
important not only for the motor-pattern switching from deposition to withdrawal but also for the maintenance of the withdrawal pattern (Fig. 10). This also indicates that initiation of the egg transport from the genital chamber into the channel between the right and left valves does not require the mechanosensory signals about the termination of withdrawal. In the locust, abdominal projection interneurons respond to the tactile stimulus of the ovipositor hair receptors (Kalogianni, 1996). The initiator interneuron inducing the withdrawal motor pattern has not been found in this study, but we also observed spiking responses of the interneurons to mechanical stimulation of the ovipositor valves (data not shown). The interneurons initiating or switching to the withdrawal motor pattern most likely receive synaptic inputs from sensory afferents of the hair sensilla on inside of the tip of the dorsal valve.

Furthermore, it has been reported that nitric oxide (NO) can regulate the frequency of egg-laying movements via activating sGC/cGMP in the locust (Newland and Yates, 2007). NO/cGMP pathway is involved in the pattern-generating network for various types of rhythmic motor activities in invertebrates and vertebrates, such as feeding and ventilation in locust (Rand et al., 2008; Armstrong et al., 2009), feeding in mollusk (Kobayashi et al, 2000; D’yakonova and D'yakonova, 2008), feeding in crab (Stein et al., 2005) and swimming of Xenopus tadpole (McLean and Sillar, 2000). It is notable that both NO-synthase (NOS) containing neurons and NO-induced cGMP-elevated neurons have also been found within the cricket TAG (Aonuma et al., 2008). The physiological role of NO/cGMP pathway in the cricket TAG has been unknown, but it is possible that the NO/cGMP signaling may play a role in switching or alternation of the oviposition motor patterns. Pharmacological studies using NOS inhibitors, NO scavengers and NO donors would account for the modulation mechanism of multiple motor patterns that are sequentially excluded during a series of oviposition behavior. 


\section{Acknowledgements}

We thank Dr. John P. Miller (Montana State University) for his helpful comments on the manuscript. We also thank Mrs. Yoshiko Sato-Kobashi for additional experiments and comments on EMG recordings. 


\section{References}

Akaike, H., 1974. A new look at the statistical model identification. IEEE Transaction on Automatic Control 19, 716-723.

Aonuma, H., Kitamura, Y., Niwa, K., Ogawa, H., Oka, K., 2008. Nitric oxide-cyclic guanosine monophosphate signaling in the local circuit of the cricket abdominal nervous system. Neuroscience 157, 749-761.

Armstrong, G.A., Rodgers, C.I., Money, T.G., Robertson, R.M. 2009. Suppression of spreading depression-like events in locusts by inhibition of the NO/cGMP/PKG pathway. Journal of Neuroscience 29, 8225-8235.

Baba, Y., Hirota, K., Yamaguchi, T., 1991. Morphology and response properties of wind-sensitive non-giant interneurons in the terminal abdominal ganglion of crickets. Zoological Science 8, 437-445.

Baba, Y., Hirota, K., Shimozawa, T., Yamaguchi, T., 1995. Differing afferent connections of spiking and nonspiking wind-sensitive local interneurons in the terminal abdominal ganglion of cricket Gryllus bimaculatus. Journal of Comparative Physiology A 176, 17-30.

Bacon, J.P., Altman, J.S., 1977. A silver intensification method for cobalt-filled neurones in wholemount preparations. Brain Research 138, 359-363.

Berkowitz, A., 2002. Both shared and specialized spinal circuitry for scratching and swimming in turtles. Journal of Comparative Physiology A 188, 225-234

Briggman, K.L., Kristan WB Jr. 2006. Imaging dedicated and multifunctional neural circuits generating distinct behaviors. Journal of Neuroscience 26, 10925-10933.

Briggman, K.L., Kristan W.B Jr., 2008. Multifunctional pattern-generating circuits. Annual Review of Neuroscience 31, 271-294. 
Carrow, G.M,, Cabeza, R.De.J., Flores, G., 1982. Isolation of the abdomen releases oviposition behaviour in females of the cricket, Acheta domesticus. Journal of Insect Physiology 28, 401-404.

Dambach, M., Igelmund, H., 1983. Das Ei-Ablageverhalten von Grillen (Saltatoria: Grylloidea). Entomologia Generalis 8, 267-281.

Delcomyn, F., 1980. Neural basis of rhythmic behavior in animals. Science 210, 492-498.

D'yakonova, T.L., D'yakonova, V.E. 2008. Modification of the effects of glutamate by nitric oxide (NO) in a pattern-generating network. Neuroscience and Behavioral Physiology 38, 407-13.

Englemann, F. 1970. The physiology of insect reproduction. Pergamon Press, New York, Oxford.

Facciponte, G., Lange, A.B., 1992. Characterization of a novel central pattern generator located in the VIIth abdominal ganglion of Locusta. Journal of Insect Physiology $38,1011-1022$

Facciponte, G., Lange, A.B., 1996. Control of the motor pattern generator in the VIIth abdominal ganglion of Locusta: Descending neural inhibition and coordination with the oviposition hole digging central pattern generator. Journal of Insect Physiology 42, 791-798.

Faraway, J.J., 2006. Extending the linear model with R: Generalized linear, mixed effects and nonparametric regression models. Chapman \& Hall/CRC, Boca Raton.

Hennig, R.M., 1990. Neuronal control of the forewings in two different behaviours: Stridulation and flight in the cricket, Teleogryllus commodus. Journal of Comparative Physiology A 167, 617-627. 
Gras, H., Kohstall, D., 1998, Current injection into interneurones of the terminal ganglion modifies turning behaviour of walking crickets. Journal of Comparative Physiology, A., 182, 351-361.

Grillner. S.. 2006. Biological pattern generation: the cellular and computational logic of networks in motion. Neuron 52, 751-766.

Jacobs, G.A., Murphey, R.K., 1987, Segmental origins of the cricket giant interneuron system. Journal of Comparative Neurology 265, 145-157.

Kalogianni, E., 1996. Morphology and physiology of abdominal projection interneurons in the locust with mechanosensory inputs from ovipositor hair receptors. Journal of Comparative Neurology 366, 656-673.

Kiehn, O. 2006. Locomotor circuits in the mammalian spinal cord. Annual Review of Neuroscience 29, 279-306

Kobayashi. S., Ogawa, H., Fujito, Y., Ito, E. 2000. Nitric oxide suppresses fictive feeding response in Lymnaea stagnalis. Neuroscience Letters 285, 209-212.

Kristan, W.B. Jr, Wittenberg, G., Nusbaum, M.P., Stern-Tomlinson, W. 1988. Multifunctional interneurons in behavioral circuits of the medicinal leech. Experentia 44, 383-389.

Marder, E. 2000. Motor pattern generation. Current Opinion of Neurobiology $10: 691-698$.

Marder, E., Bucher D. 2001. Central pattern generators and the control of rhythmic movements. Current Biology 11, R986-996

Matsuda, R. 1976. Morphology and evolution of the insect abdomen, in: Kerkut, G.A., (Ed), International series in pure and applied biology Vol.56, Zoology Division, Pergamon Press, Oxford. 
McLean, D.L., Sillar, K.T. 2000. The distribution of NADPH-diaphorase-labelled interneurons and the role of nitric oxide in the swimming system of Xenopus laevis larvae. Journal of Experimental Biology 203, 705-713.

Miller, J.P., Selverston, A.I., 1982, Mechanisms underlying pattern generation in lobster stomatogastric ganglion as determined by selective inactivation of identified neurons. IV. Network properties of pyloric system. Journal of Neurophysiology $48,1416-1432$.

Morton, D.W., Chiel, H.J., 1994, Neural architectures for adaptive behavior. Trends in Neuroscience 10, 413-420.

Newland, P.L., Yates, P., 2007. Nitrergic modulation of an oviposition digging rhythm in locusts. Journal of Experimental Biology 210, 4448-4456.

Ogawa, H., Kawakami, Z., Yamaguchi, T. 1995. Motor pattern of the stinging response in the honeybee, Apis mellifera. Journal of Experimental Biology 189, 39-47.

Pearson, K.G., Wolf, H., 1987. Comparison of motor patterns in the intact and deafferented flight system of the locust. I. Electromyographic analysis. Journal of Comparative Physiology A. 160, 259-268.

Ramirez, J.M., Pearson, K.G., 1988. Generation of motor patterns for walking and flight in motoneurons supplying bifunctional muscles in the locust. Journal of Neurobiology 19, 257-282.

Rand, D., Gueijman, A., Zilberstein, Y., Ayali, A. 2008. Interactions of suboesophageal ganglion and frontal ganglion motor patterns in the locust. Journal of Insect Physiology 54, 854-860.

Sellier, R. 1971. L'utrastructure cuticlaire de l'oviscapte chez Gryllus campestris L. Comptes rendus hebdomadaires des séances de l'Académie des sciences. Série D: Sciences naturelles 272, 977-980. 
Snodgrass, R.E., 1933. Morphology of the insect abdomen. Part II. The genital ducts and the ovipositor. Smithsonian Miscellaneous Collections 89, 1-148.

Stein, W., Eberle, C.C., Hedrich, U.B. 2005. Motor pattern selection by nitric oxide in the stomatogastric nervous system of the crab. European Journal of Neuroscience $21,2767-2781$

Stevenson, P.A., Kutsch, W., 1987. A reconsideration of the central pattern generator concept for locust flight. Journal of Comparative Physiology A 161, 115-129.

Sugawara, T., Loher, W., 1986. Oviposition behaviour of the cricket Teleogryllus commodus: observation of external and internal events. Journal of Insect Physiology 32, 179-188.

Thompson, K.J., 1986a. Oviposition digging in the grasshopper. I. Functional anatomy and motor programme. Journal of Experimental Biology 122, 387-411.

Thompson K.J., 1986b. Oviposition digging in the grasshopper. II. Descending neural control. Journal of Experomental Biology 122, 413-425.

Venables, W.N., Ripley, B.D., 2002. Modern Applied statistics with S, fourth ed. Springer, New York.

Weimann, J.M., Meyrand, P., Marder, E. 1991. Neurons that form multiple pattern generators: identification and multiple activity patterns of gastric/pyloric neurons in the crab stomatogastric system. Journal of Neurophysiology 65, 111-122. 


\section{Figure legend}

Fig. 1. Morphology and functional anatomy of ovipositor muscles. (A) Ovipositor muscles and basal cuticular plates of the ovipositor were drawn from a dorsal dissection. Four pairs of muscles; M271, M273, M285, M288 are attached to the dorsal gonapophyses, which are dorsal apodemes of the dorsal valve. A pair of muscles, M272 is attached to the ventral gonapophyses. The attachment points of M271 and M288 to the dorsal gonapophyses were indicated by shading. Another attachment site of these ovipositor muscles are on the 7 th or 8 th tergum. (B) Schematic diagram of lateral view of the abdomen, which shows the mechanism for the antero-posterior movement of the dorsal valve. Muscle attachments to the tergum are indicated by a shaded circle, and attachments to the apodemes of the ovipositor valve are shown as a filled triangle. Arrows indicate the muscle contractions and the movements of gonapophyses and ovipositor valves. Contraction of M288 pulls the dorsal gonapophosis and protracts the dorsal valve (B2). The dorsal gonapophysis is then pulled back by contraction of M271, and the dorsal valve is retracted to return the basal position (B1). DG, dorsal gonapophysis; GC, genital chamber; VG, ventral gonapophysis; $1 \mathrm{Vlv}$, ventral valve; $3 \mathrm{Vlv}$, dorsal valve. A, anterior; $\mathrm{P}$, posterior; $\mathrm{M}$, medial; L, lateral; D, dorsal; V, ventral.

Fig. 2. Innervations to the ovipositor muscles and morphology of the ovipositor motoneurons. (A) Diagram of the terminal abdominal ganglion, and the ventral roots of 8 th and 9th segmental nerve-cords (8vpn and 9vpn). The abbreviations presented at end of the nerve branches signify the effectors that are innervated by each nerve. (B) Drawing from dorsal (left drawings) and lateral (right drawings) aspects of the 
ovipositor motoneurons filled with nickel chloride in the terminal abdominal ganglion. (B1) The motoneurons supplying M271 and M273. (B2) The motoneurons supplying M272. Scale bar: $100 \mu \mathrm{m}$.

Fig. 3. Motor program in the rhythmic movement of ovipositor valves. (A) Electromyograms (EMGs) were simultaneously recorded from different pairs of ovipositor muscles in tethered and dissected preparation. Each pair of recordings were obtained from different animals. Scale bar: $500 \mathrm{~ms}$. (B) Phase relationships between right and left ovipositor muscles. The motor pattern was predicted from the EMG recordings. Black bars indicate the burst duration in which a given muscle exhibits the single (or rarely dual) discharge(s). Right and left homologous muscles contracted in antiphase during the rhythmic movements of ovipositor valves.

Fig. 4. Oviposition motor patterns during egg-laying behavior. (A) Electromyograms recorded from Left M271 and Right M288 in a freely-moving cricket during the penetration (top), egg-deposition (middle) and withdrawal steps (bottom). All recordings were obtained from a single animal which performed a sequence of egg-laying behavior. Scale bar: $500 \mathrm{~ms}$. (B) Relationships between the cycle period of successive electrical discharges (T1 in A) and the burst duration of each discharge (T2 in A) measured from EMG recording of M271 at three different steps. Open triangles indicate the data in the penetration pattern. Open circles indicate the data in the deposition pattern. Cross marks indicate the data in the withdrawal pattern. Lines indicate the mean value of burst duration predicted from the cycle period by GLM analysis for each motor pattern: solid line for penetration, broken line for deposition, dotted line for withdrawal. Gray shaded areas indicate the predicted area into which 
$50 \%$ of data fall assuming that burst duration follows gamma distribution. The set of data in (B) was obtained from 280 cycles of rhythmic activities for all motor patterns, which were recorded in a few trails in 6 animals that performed the egg-laying behavior.

Fig. 5. Spiking activities of interneurons related to initiation of the rhythmic activity in ovipositor muscles. In Figs 5A, 5B, 6A, 6B, 7A and 7B, the right drawings show the morphology of the interneurons in a TAG outline (dorsal- and posterior view). Above each intracellular recording a reference EMG obtained from M271 is presented. (A) Recordings obtained from the local spiking interneuron $8 \mathrm{~V}-1$ (LSI-8V-1). Firing frequency of spontaneous spikes increased during rhythmic bursting of M271 (A1). When the spontaneous firing was suppressed by a hyperpolarizing current injection, the action potentials were evoked preceding initiation of the rhythmic motor pattern (A2). (B) Recordings obtained from the non-giant spiking interneuron 17 (NGI-17), which projects an axon into the connective nerve-cord contra-lateral to its soma. A depolarizing current pulse (indicated as a monitorial trace in bottom) caused the rhythmic activity in M271. Horizontal scale bars: $500 \mathrm{~ms}, 100 \mu \mathrm{m}$. Vertical scale bars: $10 \mathrm{mV}$. (C) Relationships between the cycle period and the burst duration in the motor patterns generated after excitation of the 'initiator interneurons'. Filled circles in $\mathrm{C} 1$ indicate the data from the spontaneously-evoked patterns in LSI-8V-1, shown in A. Filled circles in C2 the data from the pattern caused by depolarizing current pulse to NGI-17 shown in B (2). The other plots (open triangles, open circles and cross marks) indicate the data measured in freely-moving preparations during the oviposition behavior, and the lines indicate mean value of the burst duration predicted from the cycle period by 
GLM, which are the same lines shown in Fig. 4B: solid line for penetration, dotted line for deposition, dashed line for withdrawal.

Fig. 6. Activity of the oscillator interneuron, which synchronized with the rhythmic bursting of ovipositor muscle. Intracellular recordings were obtained from a local spiking interneuron 9L-4 (LSI-9L-4) shown in the right drawing. (A) Recordings of rhythmic activity induced by cutting the connective nerve cords. (B) Recording of rhythmic oscillation of the membrane potential during a hyperpolarizing current injection into LSI-9L-4 to suppress the bursting action potentials. Although rhythmic contraction of the M271 was stopped, the membrane potential of LSI-9L-4 continued to oscillate. Arrowheads in B indicate the rhythmic depolarization of LSI-9L-4. Horizontal scale bars: $200 \mathrm{~ms}, 100 \mu \mathrm{m}$. Vertical scale bars: $10 \mathrm{mV}$.

Fig. 7. Activity of the oscillator interneurons that showed biphasic depolarization synchronized with the rhythmic bursting of the ovipositor muscle. (A) Recordings obtained from the local spiking interneuron 10-1 (LSI-10-2) shown in the upper-right drawing. A1 and A2 were recorded from different animals exhibiting distinct motor rhythms. (B) Recording obtained from local spiking interneuron 9V-4 (LSI-9V-4) shown in the lower-right drawing. Arrowheads in A2 and B indicate the two distinct components of the biphasic depolarization. Horizontal scale bars: $200 \mathrm{~ms}, 100 \mu \mathrm{m}$. Vertical scale bars: $10 \mathrm{mV}$. (C) Relationships between the cycle period and the burst duration in the motor patterns of M271 shown in A1 and A2. Filled circles in C1 indicate the data from the recording in $\mathrm{A} 1$, in which the monophasic depolarization appeared in LSI-10-2. Filled circles in C2 indicate the data from A2, in which the biphasic depolarization appeared in LSI-10-2. The other plots (open triangles, open 
circles and cross marks) indicate the data measured in freely-moving preparations during the oviposition behavior, and the lines indicate mean value of the burst duration predicted from the cycle period by GLM, which are the same lines shown in Fig. 4B: solid line for penetration, dotted line for deposition, dashed line for withdrawal. (D) Distribution of the cycle period obtained by GLM analysis to categorize the motor pattern observed with monophasic depolarization in LSI-10-2. Abscissa indicates values of the cycle period, and ordinate indicates the predicted density. We assumed that the distribution follows gamma distribution. Solid curve indicates the distribution predicted by 'one distribution model', and broke curve indicates that predicted by 'two distribution model'. Open circles indicate actual data of the cycle period in the motor pattern shown in A2 (upper) and in three motor patterns recorded in free-moving preparation (lower).

Fig. 8. Mechanoreceptive hair sensilla located on inner side of the ovipositor valve. (A) Central projection of afferent fibers from dorsal ovipositor-valve in the TAG (dorsal and lateral views). Scale bar: $100 \mu \mathrm{m}$. (B), (C) Scanning electron micrograph of the hair plates. $\mathrm{B}$ is the medial view of the whole hair plate, and $\mathrm{C}$ indicates external structure of individual hair-sensilla at different positions at higher magnification. Scale bars: $100 \mu \mathrm{m}$ in $\mathrm{B}, 10 \mu \mathrm{m}$ in $\mathrm{C}$.

Fig. 9. The hair sensilla detecting egg-deposition are required for switching the oviposition motor patterns. (A) Responses of the hair plates on the inner side of the dorsal valve to deflection of the hair shafts. Afferent spikes were recorded extracellularly from the afferent nerve in the dorsal valve during continuous deflection of the hairs in 3 different directions. The bar below the recording trace 
indicates the direction and duration of the deflection stimulus. Right inset is a schematic diagram showing examples of the monitor of mechanical stimulus, clear bar: dorsal deflection, lined bar: anterior deflection, and dotted bar: posterior deflection. Traces in A1 and A2 correspond to selected regions of the recording in A, indicating the enlarged responses to the posterior- (A1) and anterior deflection (A2). Scale bars: 2 s, $200 \mathrm{~ms}$. (B) Effect of deafferentation on the EMG-pattern switching in M271 from deposition to withdrawal steps. B1 is an EMG recording in an intact freely-moving animal, in which the deposition motor pattern switched to the withdrawal pattern (shaded duration) in the few seconds preceding the egg-release from the ovipositor. B2 is a recording during the period after the afferent fibers in the dorsal valves were cut off. B3 is a recording during the period after the hair sensilla located on inner side of top of the dorsal valves were removed. Dashed line indicates the timing of egg release from ovipositor in each experiment, which was observed by eye and manually marked on the physiological recordings using an electronic switch. In B2 and B3, the motor pattern did not switch from deposition to withdrawal even when the egg was released. Scale bar: $1 \mathrm{~s}$.

Fig. 10. Schematic model of the neural mechanism for generating and switching the motor patterns of the rhythmic movement of the ovipositor valves. The oviposition 'release' command from higher centers, such as the brain or other ganglia, induces rhythmic activity of the oviposition pattern generator via 'initiator interneurons'. The pattern generating networks partly share 'oscillator interneurons' and are rewired for generating different motor patterns in penetration, egg-deposition and withdrawal steps. Different motor outputs drive rhythmic and sequential contraction of the ovipositor muscles. Sensory afferents from hair sensilla detect egg-deposition and 
trigger the switching from deposition motor pattern to withdrawal pattern.

Supplemental Fig. 1. Activity of another type of initiator interneuron identified. (A) Intracellular recordings were obtained from a local spiking interneuron LSI-8L-2 (LSI-8L-2) shown in the right drawing. Spontaneous spikes were increased in frequency preceding the rhythmic discharge of M271. (B) When the spontaneous firing was suppressed by a hyperpolarizing current injection, the rhythmic contraction did not occur in M271. Horizontal scale bars: $200 \mathrm{~ms}, 100 \mu \mathrm{m}$. Vertical scale bars: $10 \mathrm{mV}$.

Supplemental Fig. 2. Activity of 6 types of oscillator interneurons identified. (A-C) Intracellular recordings were obtained from non-giant interneurons 18, 19 and 20 (NGI-18, 19 and 20), respectively. (D-E) Intracellular recordings were obtained from local spiking interneurons 8L-3, 9V-5 and 9V-6 (LSI-8L-3, 9V-5 and 9V-6), respectively. These interneurons showed rhythmic bursting and/or oscillation in membrane potential, which synchronized with the discharge of M271. Horizontal scale bars: $200 \mathrm{~ms}, 100 \mu \mathrm{m}$. Vertical scale bars: $10 \mathrm{mV}$. 
Table 1. Parameters characterizing the motor patterns recorded at different steps, penetration, egg-deposition and withdrawal, which are indicated in the leftmost column. The next two columns give the cycle period of successive electrical discharges and the duration of each discharge of M271 (mean $\pm \mathrm{SD}$, in ms). The next two columns give the AIC values of the model (1)-I and the model (1)-II (see Materials and Methods). The next column gives the equation with predicted parameters. The final column gives the sample sizes that are the numbers of cycles and of animals. 


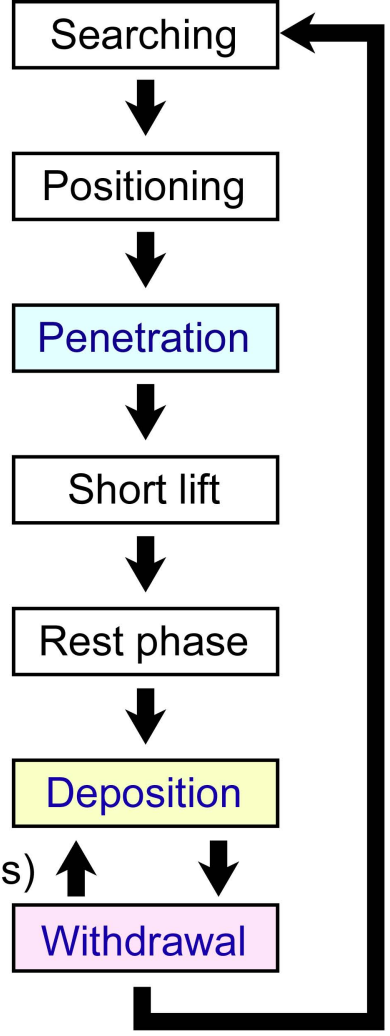

Sequence of steps in egglaying behavior in cricket
Neural mechanism for generating and switching the oviposition motor patterns 
A Oviposition motor patterns of cricket shift according to behavioral steps.

$\Delta$ Initiator and oscillator interneurons were involved in oviposition motor pattern.

A Network of oscillators were rewired for motor pattern shift.

A Afferent signal from sensory hairs on ovipositor valve triggers motor pattern shift. 

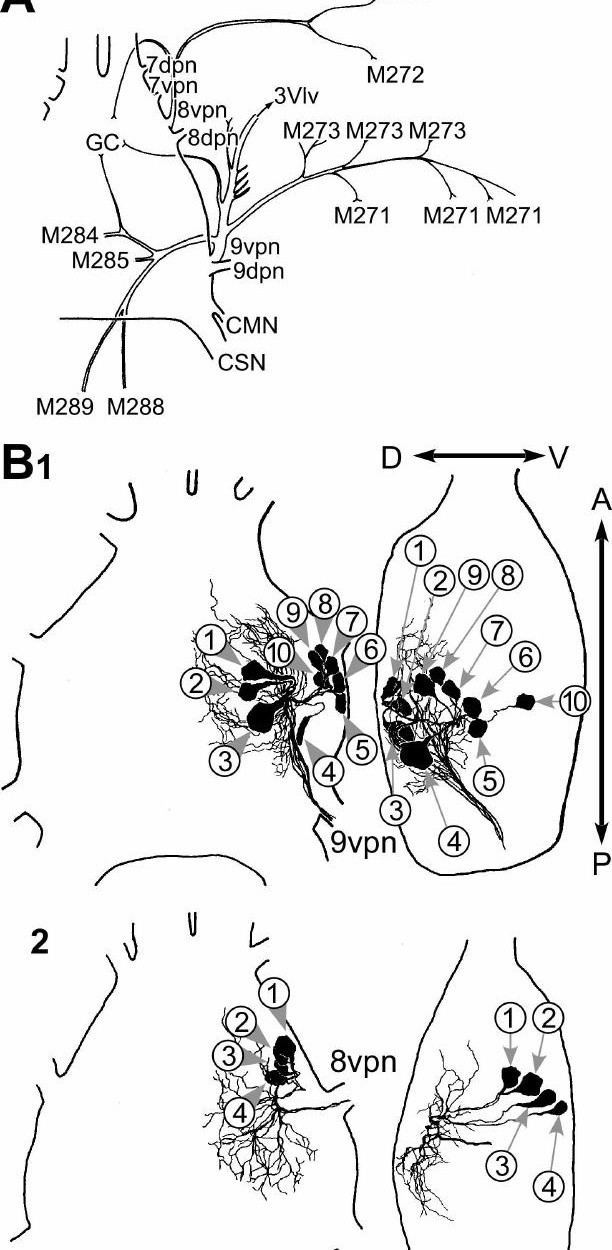

V

7

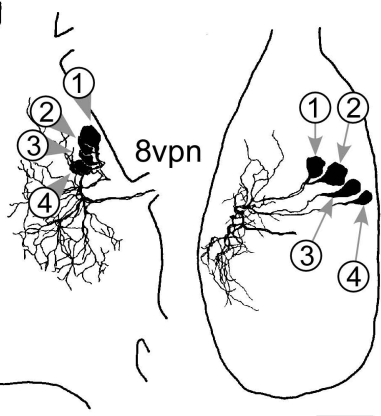




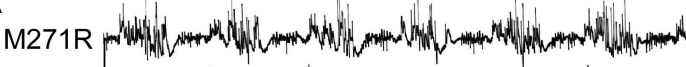

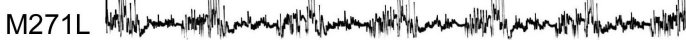
M271R

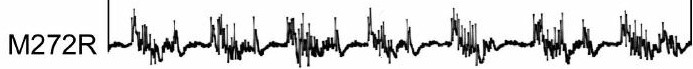

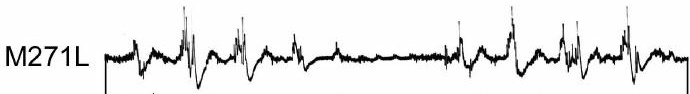

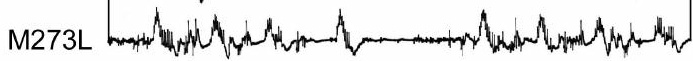
M271L M285L

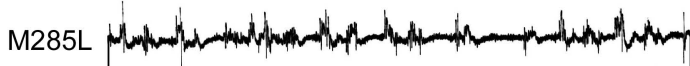
M288L

B

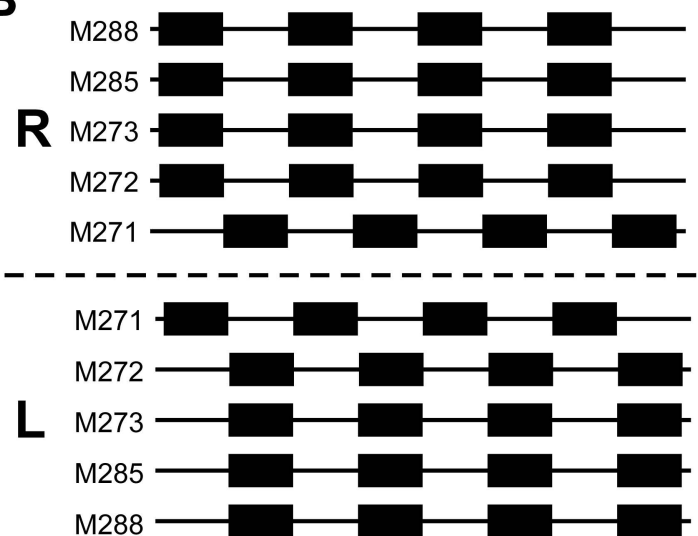




\section{Penetration}

M271L Hom

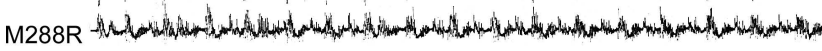

\section{Egg-deposition}

M271L

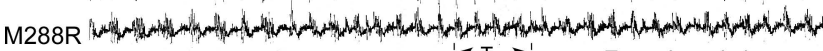

\section{Withdrawal} $-T_{1} \rightarrow$

ithdrawal

$T_{1}$ : cycle period

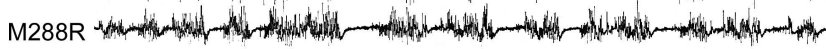

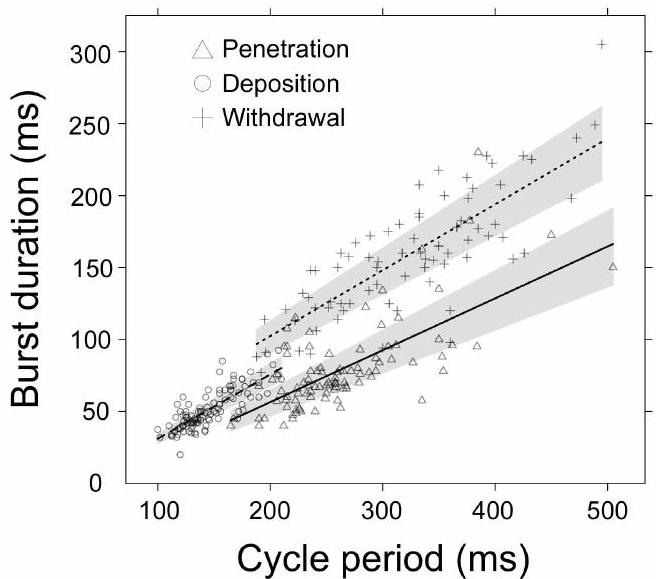




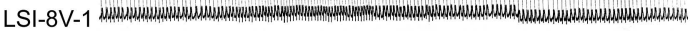

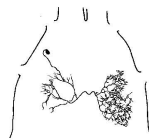

2
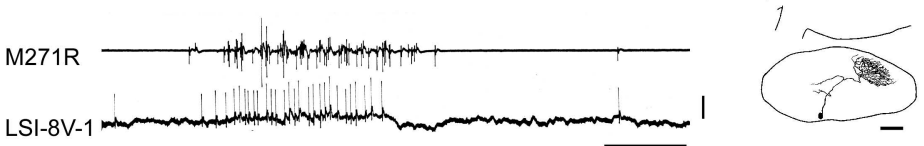

B

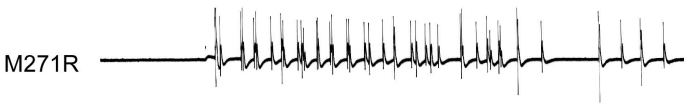

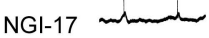

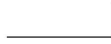

C
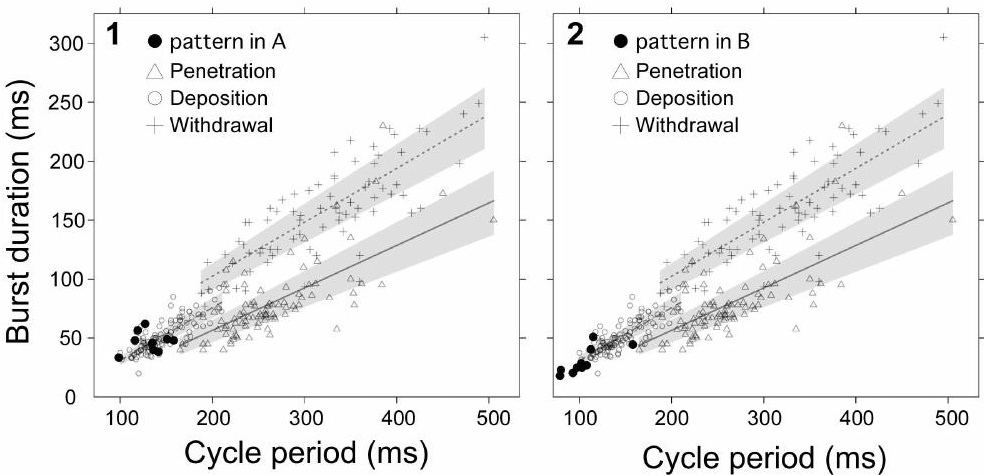
M271R -

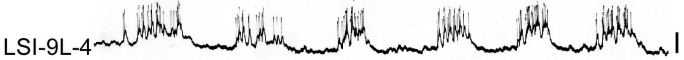

M271R بs LSI-9L-4 I I I 


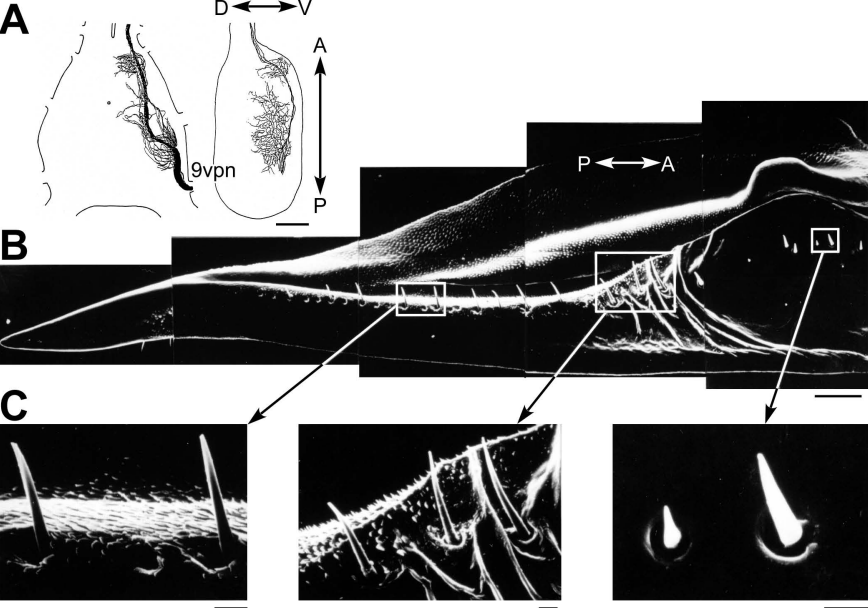




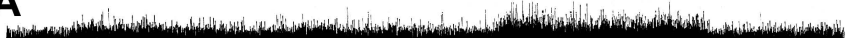

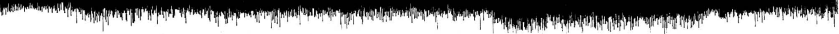

$\rightarrow 1:-2 \rightarrow$

What of

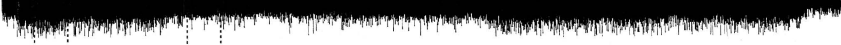

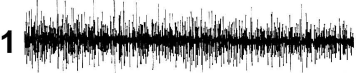

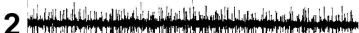

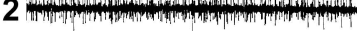

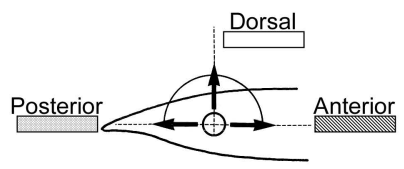

B

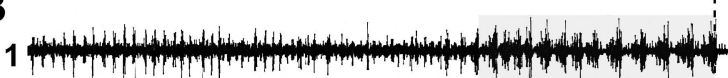

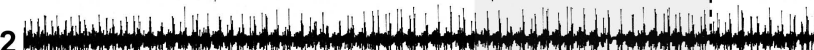

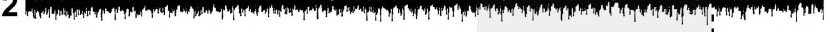

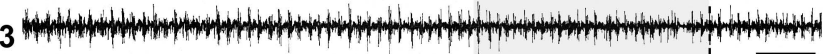




\section{Higher command center}

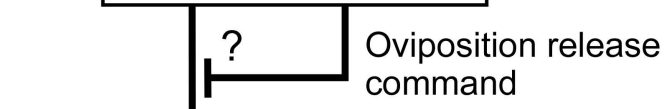

TAG

Initiator

Oviposition CPG

Penetration Deposition Withdrawal
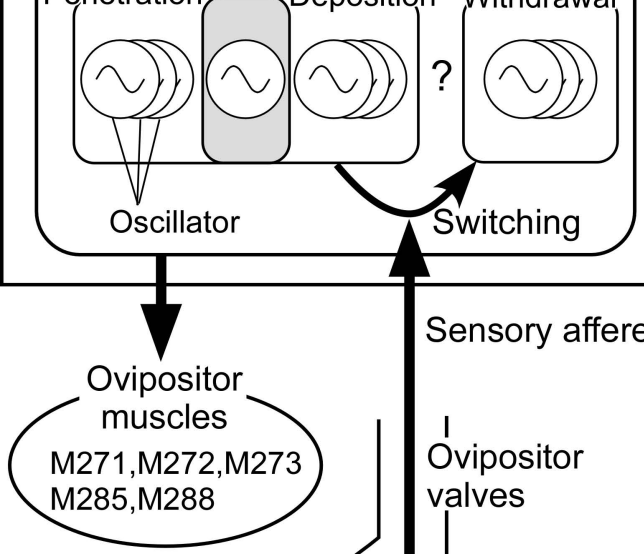

Sensory afferents 
$\mathbf{A}$

M271R

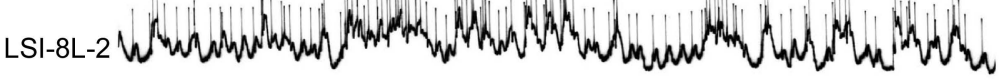

B

M271R

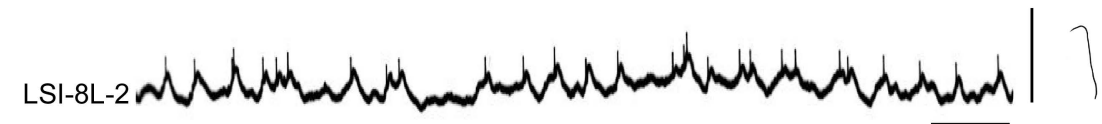

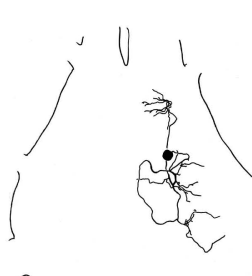




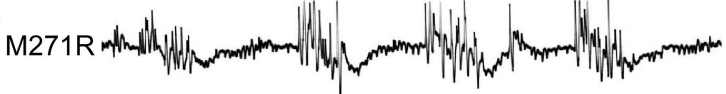
NGI-18
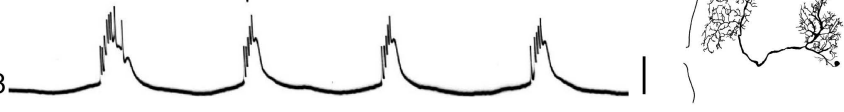

B

M271R
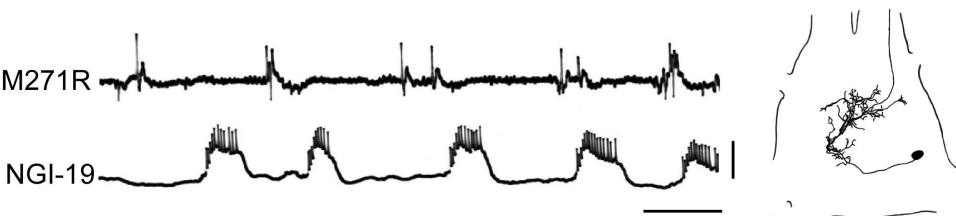

C

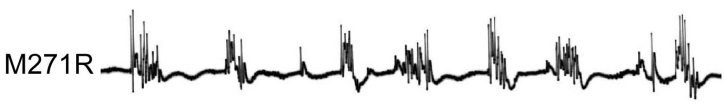

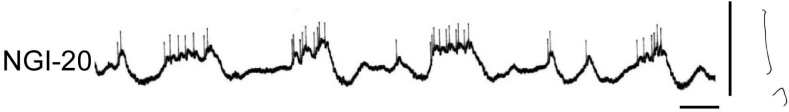

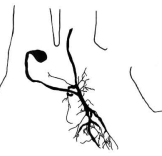

D

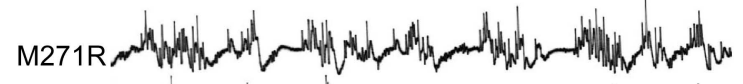

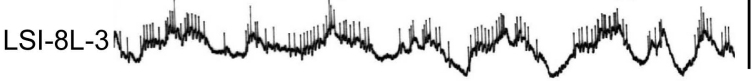

E

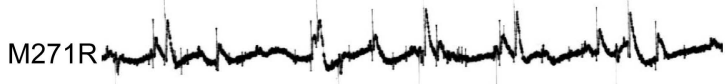
M271R

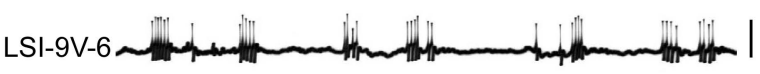

